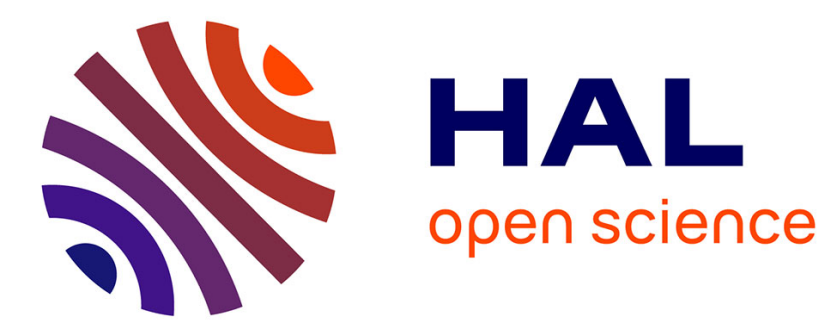

\title{
L'ÉCOPOÉSIE BRITANNIQUE AU DÉBUT DU XXIE SIÈCLE
}

Joanny Moulin

\section{To cite this version:}

Joanny Moulin. L'ÉCOPOÉSIE BRITANNIQUE AU DÉBUT DU XXIE SIÈCLE. Etudes Anglaises, 2007, Poésie contemporaine (1985-2005): Grande-Bretagne et Irlande, 60 (3), pp.317-329. hal01076360

\section{HAL Id: hal-01076360 \\ https://hal.science/hal-01076360}

Submitted on 21 Oct 2014

HAL is a multi-disciplinary open access archive for the deposit and dissemination of scientific research documents, whether they are published or not. The documents may come from teaching and research institutions in France or abroad, or from public or private research centers.
L'archive ouverte pluridisciplinaire HAL, est destinée au dépôt et à la diffusion de documents scientifiques de niveau recherche, publiés ou non, émanant des établissements d'enseignement et de recherche français ou étrangers, des laboratoires publics ou privés. 


\title{
L'ÉCOPOÉSIE BRITANNIQUE AU DÉBUT DU XXIE SIÈCLE
}

\author{
Joanny Moulin
}

Klincksieck | Études anglaises

$2007 / 3$ - Vol. 60

pages 317 à 329

\section{ISSN 0014-195X}

Article disponible en ligne à l'adresse:

http://www.cairn.info/revue-etudes-anglaises-2007-3-page-317.htm

Pour citer cet article :

Moulin Joanny, « L'écopoésie britannique au début du XXle siècle »,

Études anglaises, 2007/3 Vol. 60, p. 317-329.

Distribution électronique Cairn.info pour Klincksieck.

(c) Klincksieck. Tous droits réservés pour tous pays.

La reproduction ou représentation de cet article, notamment par photocopie, n'est autorisée que dans les limites des conditions générales d'utilisation du site ou, le cas échéant, des conditions générales de la licence souscrite par votre établissement. Toute autre reproduction ou représentation, en tout ou partie, sous quelque forme et de quelque manière que ce soit, est interdite sauf accord préalable et écrit de l'éditeur, en dehors des cas prévus par la législation en vigueur en France. II est précisé que son stockage dans une base de données est également interdit. 
Joanny MOULIN

\section{L'écopoésie britannique au début du XXI ${ }^{\mathrm{e}}$ siècle}

Cet article tente d'explorer et de définir le versant britannique du mouvement international contemporain qu'est l'écopoésie. Un bref examen de la notion d'écocritique, considérée comme un moment post-déconstructionniste de la théorie et de la critique littéraire, en vient à réaliser que plusieurs de ses arguments sont des reprises de considérations anciennes, dont le modernisme et le postmodernisme s'étaient détournés. À plus d'un titre, le discours de l'écopoésie et de l'écocritique est une résurgence et une reprise de l'idéologie romantique, pour lequel la figure de William Wordsworth revêt une importance particulière. Dans un certain sens, c'est une alternative au postmodernisme, qui trouve des soutiens théoriques chez des penseurs situés en marge du post-structuralisme, continuant ainsi une tradition séculaire d'écrivains qui portèrent un regard critique sur le rationalisme matérialiste occidental.

His article is an attempt to explore and assess the British side of the contemporary international movement of ecopoetry. It begins with a brief examination of ecocriticism, considered as a post-deconstruction moment of literary theory and criticism, coming to realise that several of its main lines of argument are revisions of previous considerations, that modernism and postmodernism had turned away from. In several ways, the discourse of ecopoetry and ecocriticism is a resurgence and a revaluation of the romantic ideology, for which William Wordsworth is a figure of particular importance. It is in some respect an alternative to postmodernism, finding theoretical support in thinkers in the margins of poststructuralism, thus continuing a long-standing tradition of writers who looked with critical eyes on Western materialist rationalism.

Le terme écopoésie (ecopoetry) provient du vocabulaire de l'écocritique (ecocriticism), une école de théorie littéraire d'origine américaine dont l'épicentre est l'ASLE (Association for the Study of Literature and Environment), fondée en 1992 à Reno (Nevada) lors d'une réunion spéciale de la Western Literature Association, et qui tient des congrès bisannuels depuis 1995. Son organe de publication est la revue ISLE (Interdisciplinary Studies in Literature and Environment), dont un équivalent britannique est la revue Green Letters. Ce mouvement de la critique contemporaine fait montre d'un dynamisme qu'attestent les différents courants qui le composent 
et les quelques controverses qui le secouent. Il s'agit en réalité d'un faisceau de discours critiques divers. S'il fallait ne mentionner pour commencer que deux auteurs et leurs ouvrages les mieux connus, qui puissent être des ambassadeurs très reconnus de l'écocritique, il conviendrait de citer respectivement deux noms. Aux États-Unis, Lawrence Buell, professeur de littérature américaine à l'Université de Harvard, auteur entre autres de The Environmental Imagination: Thoreau, Nature Writing, and the Formation of American Culture (1995) et de The Future of Environmental Criticism (2005). Au Royaume-Uni, Jonathan Bate, professeur d'études shakespeariennes et de littérature de la Renaissance à l'Université de Warwick, auteur de Romantic Ecology: Wordsworth and the Environmental Tradition (1991) et de The Song of the Earth (2000). L'écocritique ne s'intéresse pas principalement à la nature comme thème littéraire, mais c'est une critique écocentrique, qui s'interroge sur l'articulation entre l'homme et le monde (rappelons que le préfixe " éco-» provient du grec oikos, la maison, partant l'habitat), domaine dans lequel le langage, donc la littérature et particulièrement la poésie tiennent un rôle de premier plan.

Il existe donc ce que Buell appelle un «écodiscours littéraire » (2005 vii), ce qui peut se comprendre de deux manières : soit que la littérature elle-même peut être abordée comme écodiscours, soit qu'il existe de fait un certain discours critique qui aborde la littérature sous un angle écologique ou écocentrique. L'écocritique court donc le risque de n'être en fin de compte pas autre chose qu'une instrumentalisation de la littérature dans la défense de la cause écologique (voir Killingsworth et al.). Si l'écocritique n'était que cela, elle ne mériterait pas qu'on la prît en considération du point de vue littéraire. En effet, l'écocritique anglais Terry Gifford fait remarquer dans Green Voices (1995) que la "poésie verte » est vouée à l'échec en tant que poésie lorsqu'elle s'abandonne à la rhétorique de propagande (174). C'est une leçon qu'il tenait de Ted Hughes, en qui il aurait voulu voir le type même de l'écopoète britannique, mais qui lui avait fait remarquer que, chaque fois qu'il tentait de faire un poème sur un sujet écologique, c'était la poésie qui en était pour ses frais. Le rôle de l'ecopoesis, affirme Jonathan Bate, est différent : c'est un engagement imaginatif avec l'inhumain ("to engage imaginatively with the non-human " [199]). L'écodiscours littéraire est un écocentrisme.

Mais cette question prend un tour particulier dans le contexte de la littérature et de l'histoire des idées américaines. En effet, les Romantiques américains, c'est-à-dire les Transcendantalistes, ont eu un rôle fondateur dans la constitution de l'identité nationale, à laquelle participent le canon littéraire et son interprétation. Par bien des côtés, l'Amérique a fait sienne l'idéologie romantique. Dans un article pour Environmental History, Michael Cohen fait remarquer que des générations d'étudiants ont adopté les idées de leurs auteurs favoris, au point que l'acte critique s'est auréolé d'une valeur pour ainsi dire religieuse, à tout le moins chargé d'idéologie. Partant de considérations similaires, Leo Marx lança une controverse en publiant en 1999 un article sur Thoreau où il déclarait que les écocentristes 
sont les puritains du mouvement écologiste contemporain. Il entendait souligner par-là que l'écocritique n'était pas à l'abri d'une dérive vers un discours prescriptif, délivrant aux poètes des certificats d'écopoésie selon qu'ils sont plus ou moins écologiquement corrects.

Toutefois, il faut remarquer encore que le mouvement écocritique accédait à une existence institutionnelle dans le courant des années 1990, décennie marquée par la "theory war ", c'est-à-dire une résistance accrue à la théorie littéraire, d'inspiration française, issue du structuralisme et de la philosophie de la déconstruction. À bien des égards, l'écocritique a pris le contre-pied du poststructuralime et du déconstructionnisme, au point de proposer une contre-théorie, que Lawrence Buell appelle " écothéorie littéraire » (Buell 11). Il s'agit, pour Buell, de " sauver » la littérature de la distanciation que ces théories ont introduites entre le lecteur et le texte, entre le texte et le monde (Buell 6). C'est aussi la thèse soutenue par le critique américain Leonard Scigaj dans Sustainable Poetry : le langage est selon lui un instrument que l'écopoète ajuste et accorde sans cesse pour articuler son expérience originelle de la nature (Scigaj 29). Parodiant le concept de différance chez Derrida, Scigaj proposait celui de référance, pour désigner cette insistance caractéristique avec laquelle la poésie oriente le lecteur vers le référent, par-delà l'abstraction du signifié. Jakobson disait que la « fonction poétique » du langage met l'accent sur le message pour le message, la réduisant ainsi à un jeu sur le signifiant : la poésie, l'écopoésie en tout cas, fait exactement le contraire. Leonard Scigaj concevait la référance comme une modalité particulière de l'épiphanie littéraire, à laquelle l'écopoésie aboutirait par un long processus d'érosion et d'usure du mécanisme habituel de la signification, débouchant sur une révélation ontologique silencieuse, au-delà des limites du langage, comparable au «non-temps » de la pratique du Tao ou du Zen (Scigaj 38). David Gilcrest, propose dans "Regarding Silence. Cross-Cultural Roots of Ecopoetic Meditation" la notion d'épiphanie matérialiste ou taoïste, par contraste d'une part avec l'épiphanie idéaliste ou platonicienne, d'autre part avec l'épiphanie théiste ou augustinienne. Quoi qu'il en soit, l'écopoésie se caractérise par une référentialité littéraire réaffirmé, qui ne s'exerce pas nécessairement sur le mode épiphanique. Peut-être même la référentialité écopoétique s'oppose-t-elle à l'épiphanie, dans la mesure où précisément elle contredit le dualisme qu'elle présuppose.

La contradiction que l'écocritique apporte à la philosophie de la déconstruction est une réaction heideggerienne : cela revient à choisir le camp de Heidegger contre celui de Derrida. En effet, dans son discours sur la technologie («The Question Concerning Technology »), Heidegger cite Hölderlin lorsqu'il dit que "l'homme habite poétiquement la terre " ou que "l'on doit essayer d'habiter poétiquement le monde, la maison du monde ». Dans une critique de la modernité, Heidegger affirme qu'il fut un temps, dont il appelle le retour de ses vœux, où la technè ne se réduisait pas à la technologie, mais incluait la poésie, définie comme " faire-advenir du vrai dans le beau ", c'est-à-dire ce que Platon, dans le Phèdre, nomme to ekphanestaton, ce qui se révèle le plus purement (339-40). En un sens, cela revient à la 
question de l'épiphanie, mais avec cette différence que la notion essentielle d'habitation vise à abolir l'écart qu'implique l'apparaître (epi-phanein, adparescere). La poésie (c'est-à-dire en l'occurrence l'écopoésie) se voit ainsi définie comme étant précisément cet être-dans-le-monde, par lequel le sujet humain construit (poiein, c'est faire) son être par une interaction, une relation réciproque, avec son environnement naturel considéré comme habitat (oikos). L'art poétique ne consiste donc pas alors à représenter la nature, mais à éprouver sa présence.

La notion de présence revient souvent dans une certaine poésie contemporaine qu'il est juste d'appeler écopoésie pour cette raison même. En France, la poésie d'Yves Bonnefoy s'inscrit dans la même tradition. Cela se traduit souvent par un attachement amoureux à certains lieux, que Gaston Bachelard, dans la Poétique de l'espace, appelait «topophilie ». Bachelard croyait à la psychanalyse et nourrissait une fascination toute freudienne pour certains lieux dont il inclinait à donner une interprétation allégorique. Mais cette topophilie peut aussi prendre les aspects d'un amour du pays et de la nature comme pays natal. L'amour des lieux que cultive une certaine littérature vient alors aisément alimenter une rêverie nationaliste, dont n'étaient pas exempts certains Romantiques allemands, parfois qualifiés de Völkisch Romantik et que Heidegger lisait avec grand plaisir, non plus que certains philosophes comme Fichte ou Herder. Ce primordialisme est un mode de pensée souvent sous-tendu par un certain organicisme, qui imagine la culture comme émanant naturellement du sol, au même titre éventuellement que la race. Dans le domaine anglais, on retrouve ces manières de penser chez nombre d'auteurs, dont Carlyle est sans doute l'un des plus remarquables, mais aussi par exemple de façon très innocente et spontanée chez Keats.

À l'époque contemporaine, on rencontre par exemple une manière de primordialisme chez Heaney, qui l'hérite de Yeats. Mais il est intéressant de remarquer que, comme dans le cas de ces poètes irlandais entre autres, le primordialisme poétique s'est infléchi pour prendre une signification particulière dans des contextes postcoloniaux, où il conviendrait peut-être de le nommer plutôt biorégionalisme ou écolocalisme (Buell 77). On en trouve en effet certaines formes, par exemple, chez les poètes antillais Walcott ou Brathwaithe, qui tentent de faire advenir en littérature une nation caribéenne, comme le Stephen de Joyce rêvait, au terme du Portrait de l'artiste en jeune homme, de forger la conscience incréée de sa race. Ainsi, Brathwaithe a entrepris une révision de la Tempête de Shakespeare, très comparable à celle de Césaire dans Une Tempête : chacun de ces poètes recentre son poème sur Caliban, l'homme de couleur envisagé comme entretenant un lien de légitimité privilégiée avec ses îles où il entend le chant de la terre. Mais ce sont là des cas particuliers de ré-habitation (« reinhabitation ») ou de " devenir-indigène » (Buell 146), où la poésie est le truchement par lequel s'effectue une ré-appropriation mentale et culturelle de certains lieux. Ces poétiques de la ré-habitation sont à rapprocher des thèses de l'anthropologue français Marc Augé dans Non-lieu. Introduction à une anthropologie de la surmodernité (1992) : les non-lieux surmodernes 
sont précisément des espaces désinvestis de toute habitation poétique, au sens où l'entendait jadis Hölderlin.

À partir d'une remarque de Hölderlin, dans une lettre de 1799, selon laquelle le besoin artistique est un service que l'homme rend à la nature (Zimmerman 125), l'interprétation qu'en fait Heidegger revient à dire que la nature a besoin de l'homme. Une telle considération jetait la base d'une écologie qui abandonne l'anthropocentrisme, qui la condamnerait à la superficialité. En 1973, le philosophe norvégien Arne Næss avançait le concept d'écologie profonde (" deep ecology»), auquel correspond sensiblement la notion d'écosophie (écologie philosophique) proposée par Félix Guattari en 1992. L'écologie profonde va jusqu'à dire que, toutes les espèces étant écologiquement interdépendantes, aucune d'entre elles n'a davantage de droit qu'une autre à la vie. Par sa dimension éthique autant que philosophique, l'écologie profonde va souvent de pair avec l'écothéologie, selon laquelle les dégradations de la nature sont étroitement liées à certaines évolutions spirituelles et religieuses de l'homme. L'assassinat de Mère Nature, écrivait par exemple Matthew Fox dans The Coming of the Cosmic Christ (1988) est de nos jours le problème spirituel, éthique et humain prioritaire sur notre planète (144). Teilhard de Chardin et sa Noosphère tiennent en la matière une place importante, de même que l'Hypothèse Gaïa de James Lovelock. Ce sont deux visions de la terre comme un superorganisme, dans l'évolution duquel l'espèce humaine joue un rôle déterminant. La formule de Derrida selon laquelle «il n'y a pas de hors-texte " se trouve ainsi renversée, dans une révision moderne de la métaphore médiévale du liber mundi: toute la nature serait alors un texte, le poème de Dieu en somme, dont l'homme ne serait qu'un élément parmi tant d'autres. C'est dans cette optique que l'écopoète américain Gary Snyder peut intituler son recueil de poèmes choisis No Nature, signifiant par là que les productions de l'homme sont celles de la nature. De même, le journaliste Bill McKibben a pu soutenir, dans un livre intitulé The End of Nature (1989), que la nature est désormais tellement modifiée en profondeur par l'homme qu'elle n'existe pas davantage que la Frontière américaine selon F. J. Turner, c'està-dire tout au plus comme un souvenir et comme un mythe.

La notion d'écothéologie est parcourue par une question sous-jacente, soulevée dès 1967 par Lynn White Jr., professeur de littérature médiévale aux universités de Princeton et de Californie, dans un article pour Science intitulé «The Historical Roots of Our Ecologic Crisis », qui fut à l'origine d'une certaine polémique. White y soutenait en effet que l'équation occidentale entre la technologie et la science, dont il situait l'origine vers le $\mathrm{XI}^{\mathrm{e}}$ siècle et l'accomplissement vers le milieu du XIX ${ }^{\mathrm{e}}$, avait partie liée avec le christianisme, selon lui la religion la plus anthropocentrique au monde, qui non seulement a établi un dualisme qui sépare l'homme de la nature, mais encore insiste pour dire que c'est la volonté de Dieu que l'homme exploite la nature à ses fins. La science occidentale, concluait White, a été coulée dans un moule chrétien, et par conséquent le christianisme porte une écrasante culpabilité ( "Christianity bears a huge burden of guilt») dans la 
crise écologique moderne. White faisait pourtant remarquer que cette erreur est historique et non pas inhérente au christianisme, comme en témoigne la vie de saint François d'Assise, qui tenta de "déposer l'homme de son règne monarchique sur la création pour instaurer une démocratie de toutes les créatures de Dieu ". Il convient de noter au passage que cette critique du christianisme trouve un fort écho en Angleterre dans le discours poétique de Ted Hughes, qui voyait dans le christianisme réformé l'aboutissement d'une " erreur humaine » dont il situait l'origine dans la philosophie de Socrate et l'abstraction platonicienne. Les mêmes idées se retrouvent, encore une fois, dans certains commentaires de Heidegger sur les Hymnes de Hölderlin, selon lesquels c'est d'abord par le christianisme que la nature a été dépréciée et réduite au niveau d'un " monde créé » placé sous la domination surnaturelle de la grâce; c'est ensuite par la science naturelle moderne que la nature a été dénaturée et dissoute dans le commerce, la mécanisation industrielle et la technologie ${ }^{1}$.

Un tel antichristianisme n'est cependant pas une constante de l'écopoésie et on remarquera qu'il se trouve contredit assez fortement par deux Pères Jésuites : Hopkins et Teilhard de Chardin. Poète victorien dont l'œuvre ne fut véritablement découverte qu'après la Première Guerre mondiale, Hopkins avait une conception très franciscaine de l'amour christique. Ce qu'il appelait son " communisme ", au grand dam de son ami Bridges, était une manière d'écologie profonde avant la lettre, puisque c'était une extension de la charité chrétienne non seulement à tous ses frères humains, mais aussi aux moindres créatures de Dieu, comme en attestent le très connu "The Windhover », mais aussi « Binsey Poplars » ou " God's Grandeur » : "Generations have trod, have trod, have trod; / And all is seared with trade; bleared, smeared with toil;/And wears man's smudge and shares man's smell; the soil/Is bare now, nor can foot feel, being shod. » (Hopkins 66). Teilhard de Chardin, quant à lui, remarquait que " pour de multiples raisons historiques et psychologiques, la Religion du Tout s'est surtout formulée, jusqu'ici, en termes de paganisme et d'antichristianisme » (Teilhard de Chardin 1969, 81). Mais il œuvrait à réconcilier le christianisme, d'une part avec le panthéisme, d'autre part avec l'évolutionnisme. Dans "Panthéisme et Christianisme » il poursuivait en disant : "Mais il y a toujours eu des poètes. Toujours, donc, il y a eu des âmes naturellement panthéistes » (76). Sa Noosphère était ce qu'il appelait dans La Place de l'homme dans la nature "l'Humanité planétisée » (Teilhard de Chardin 1965, 106), et les liens qui rattachent l'homme à la création qu'il habite n'étaient pas autre chose pour lui que «les liaisons du corps mystique (du plérôme)"

1. "Once through Christianity, whereby nature was, in the first place, depreciated to [the level of] 'the created,' and at the same time was brought into a relation with super-nature (the realm of grace). Then [it was denatured] through modern natural science, which dissolved nature into the orbit of the mathematical order of worldcommerce, industrialisation, and in a particular sense, machine technology." (Heidegger 1995, 98) 
(Teilhard de Chardin 1969, 86). Dans un article récent, "The Trouble with Wilderness; or, Getting Back to the Wrong Nature », William Cronon analyse l'évolution américaine du concept biblique de désert ou de nature sauvage ("wilderness») en des termes auxquels Teilhard eût acquiescé sans réserve : le désert n'est plus le lieu de la tentation satanique, c'est désormais un temple sacré. Cette notion panthéiste est au centre des préoccupations de nombreux écopoètes contemporains, comme par exemple la poétesse anglaise Kathleen Raine et son concept de Temenos.

Dans le domaine de la poésie et de l'histoire des idées britanniques, il est possible de voir un cheminement comparable entre le déisme panthéiste qui caractérise le premier Wordsworth et le christianisme de sa maturité. Le long travail de Wordsworth entre le moment où en 1805 il lit The Recluse pour la première fois à Coleridge, et celui où en 1850 il publie une version profondément remaniée de son poème sous le titre The Prelude est le prototype d'une tension qui marque bon nombre de ses héritiers. Car, si l'écopoésie américaine trouve ses pères fondateurs chez Emerson et Thoreau, la britannique trouve le sien chez Wordsworth, dont l'influence n'a pas cessé de se faire fortement sentir. D'abord, point n'est besoin de redire que Wordsworth est le modèle de la poésie pastorale (" nature poetry ») : issu du renouveau pastoral et sentimental de l'époque préromantique (voir Clare, Gray, Cowper, Burns, etc.), il renouvelait la tradition de Spenser en la délivrant de son arcadisme précieux, pour lui donner une forme populaire qui lui permettait de répondre efficacement à ce que Hölderlin appelait le "besoin artistique " de son temps. Terry Gifford a bien montré, dans Pastoral (1999), la vivacité du mode pastoral dans la poésie anglaise contemporaine, mais il explique aussi de manière très convaincante, dans Reconnecting With John Muir: Essays in Postpastoral Practice (2006) ou dans son essai " Gary Snyder and the Post-pastoral » (2002), que l'écopoésie commence avec la postpastorale, c'est-à-dire au-delà non seulement de l'idéalisation que constitue la pastorale, mais aussi de l'antipastorale qui la dénonce sans pour autant la surmonter.

Or, en l'occurrence, Wordsworth semble bien occuper une positionclef, car la quadruple critique à laquelle il s'est trouvé exposé de son temps continue de structurer le champ de l'écopoésie britannique aujourd'hui. Premièrement, Blake devait lui reprocher son panthéisme même, en s'exclamant que la Déesse Nature est la femme de Satan (" "The Goddess Nature" is "Satan's Wife" » [The Laocoön]), qu'elle n'a pas de dimension surnaturelle et demeure vouée à la mort ("Nature has no Supernatural \& dissolves: Imagination is Eternity "(The Ghost of Abel plate 1). Cela renvoie à l'incompréhension toujours possible, bien que peut-être pas insurmontable, entre écopoésie et christianisme. Deuxièmement, la divergence de vue de Coleridge, qui choisit de se détourner de la référentialité à laquelle Wordsworth tenait tant, pour lui préférer une adoration tout augustinienne de son propre génie créateur et une pratique non mimétique de l'art. Troisièmement, le reproche que lui firent Shelley et Byron de s'être détourné du panthéisme et de l'esprit philosophique révolutionnaire de ses 
premières années, pour retomber dans le giron de la religion chrétienne et d'opinions politiques trop conservatrices à leur goût : cela préfigurait le dualisme gauche-droite de l'écopoésie actuelle. Quatrièmement, l'accusation d'égotisme que devait lui adresser Keats - " the poetical Character itself [must be] distinguished from the Wordsworthian or egotistical sublime » (Letter to R. Woodhouse, 27 Oct. 1818) - c'est le point de départ d'un discours écopoétique qui conteste le concept empiriste ou cartésien de sujet. Les deux premiers moments de cette problématique (critique du christianisme et référentialité) ont été abordés précédemment; restent à examiner les deux suivants (implications politiques et question du sujet).

Il y a de nombreuses ressemblances entre Wordsworth et Rousseau, en dépit du fait que le premier ouvre en Angleterre la période romantique qui se définit comme une critique de la philosophie des Lumières, dont le second est un insigne représentant. Comme le soulignait Jean Starobinski dans JeanJacques Rousseau : la transparence et l'obstacle (1976), dans les Discours et dans l'Émile Rousseau insiste sur le fait que la société nie la nature et la voile. Il opère ainsi comme une révision du platonisme qui se trouve déjà chez Blake, pour qui la nature est le monde mensonger de Urizen, le mauvais Dieu, qui masque à la vision ordinaire une vérité qu'il incombe à la poésie de dévoiler, par une corrosion semblable à celle de l'acide du graveur. Chez Rousseau, c'est la société qui masque la vérité de la nature, que la philosophie a pour mission de retrouver. Dans le Discours sur l'origine de l'inégalité, lorsque Rousseau en vient à considérer l'homme "par le côté métaphysique et moral », il dit que l'homme, comme l'animal, n'est pour lui rien d'autre " qu'une machine ingénieuse, à qui la nature a donné des sens pour se remonter elle-même " (Rousseau 170-71), avec cette seule différence que l'animal est guidé par l'instinct, c'est-à-dire la nature, tandis que l'homme se guide lui-même par un acte de liberté, courant par là le risque de se tromper à ses propres dépens. Il semble donc possible de trouver chez Rousseau les prémices d'un discours écologique de gauche, auquel fait écho, par exemple, le titre de l'ouvrage de Michel Serres, Le Contrat Naturel (1990), qui prétend doucement étendre la notion de contrat social à l'ensemble de l'environnement naturel de l'homme. Sur un tel discours viendront aisément s'articuler les notions de « justice écologique » ("environmental justice ») et celle de «socialisme écologique » ou " écologie sociale ", qui intègrent à leur propos le refus du capitalisme, selon une ligne de pensée assez proche des arguments développés, par exemple, par Max Weber dans L'Éthique protestante et l'Esprit du capitalisme (1905). Ainsi, sur l'aile gauche de l'écocritique, se trouvent les héritiers de quelques grands précurseurs, au nombre desquels Leo Marx, l'auteur de The Machine and the Garden: Technology and the Pastoral Ideal in American Culture (1964) ou Raymond Williams, dont l'ouvrage The Country and the City (1973) fit un peu figure d'écocritique avant la lettre. Cette tradition intellectuelle a tendance à trouver les Romantiques idéologiquement suspects. Ainsi, par exemple, dans Wordsworth: the Sense of History (1989), Alan Liu dénonce la dévotion de Wordsworth pour la nature comme relevant d'opinions politiques conservatrices. En effet, il ne serait pas faux de dire 
que, jusqu'à une époque récente où se fait sentir davantage l'influence américaine, l'écopoésie britannique s'est caractérisée par une inflexion idéologique marquée à droite.

Le lien entre discours poétique et pensée de droite se fait assez naturellement, si l'on peut dire, par le biais du primordialisme, dont il a été question précédemment. Inutile de rappeler comment l'ordre naturel peut aisément être pris pour garant de l'ordre établi, et comment la théorie de l'évolution, qui a grandement contribué à permettre le développement de l'écologie (le terme même fut inventé par le darwiniste allemand Ernst Haeckl en 1866), a par ailleurs donné lieu à différentes formes de darwinisme social justifiant la domination du plus faible par le plus fort. Dans Le Nouvel ordre écologique (1992), Luc Ferry s'intéresse à ce versant de l'écodiscours, dont il nomme écofascisme les versions extrémistes, et fait remarquer que l'idéologie nazie était fortement imprégnée d'un certain écologisme. Il est également aisé de voir comment la pensée de Heidegger établit un lien entre l'écologie et le national-socialisme, qu'il ne renia jamais, et auquel il semble établi qu'il adhéra par manière de refus de la civilisation industrielle technologique, dont les USA et l'URSS étaient deux expressions rivales mais fondamentalement équivalentes à ses yeux. Certains points communs entre l'écosophie et la psychologie des profondeurs d'un Jung, par exemple, vont dans le même sens. Dans la littérature anglaise, on pourrait trouver certaines possibilités de dérives comparables chez des écrivains comme Wyndham Lewis ou D. H. Lawrence, ou bien encore dans la fascination pour la vie animale qui caractérise un Henry Williamson, le célèbre auteur de Tarka la loutre (1927). Même s'il a toujours pris soin de prendre ses distances avec Williamson, Ted Hughes a souvent prêté le flanc à de semblables critiques, par sa célébration de la violence animale, son néochamanisme et l'anti-humanisme d'inspiration nietzschéenne de son discours poétique. Seamus Heaney s'est lui-même exposé à des reproches de cet ordre au moment de la publication de North (1975), de la part de certains critiques qui n'étaient pas tout à fait assez sûrs de bien percevoir l'ironie dénonciatrice de certains de ses textes.

Néanmoins, la ligne de démarcation entre écologie de gauche et écologie de droite, plus exactement la raison principale de la réception problématique de l'écopoésie britannique par la critique de gauche, tient à ce qu'elle est toujours suspecte de revenir à un discours anti-moderne et antiLumières. Entre Wordsworth et Rousseau subsiste une différence essentielle. Car Rousseau s'en tient à un point de vue mécaniste qui est celui de Descartes et sa transcendance est celle de la Raison. Mais ce qui fait de Wordsworth le père de l'écopoésie britannique, jouant en cela un rôle historique comparable à celui de Thoreau et d'Emerson en Amérique, c'est qu'il est le poète panthéiste de la présence. Toutefois, cette notion renvoie davantage au Sublime de Burke qu'au rationalisme de l'idéologie française : «I have felt / A presence that disturbs me with the joy / Of elevated thoughts; a sense sublime / Of something far more deeply interfused, / Whose dwelling is the light of setting suns, / And the round ocean, and the living air, / 
and the blue sky, and in the mind of man » («Tintern Abbey »). À tort ou à raison, cette notion de présence a été identifiée comme étant par excellence ce «signifié transcendantal » dont la philosophie de la déconstruction a voulu tourner la page. La critique faite par Keats contre le prétendu " égotisme » de Wordsworth (bien qu'elle portât plutôt sur le projet autobiographique du Prélude et non pas tant sur la présence), a trouvé sa traduction dans le vocabulaire de la théorie post-structuraliste : Wordsworth fut jugé "logocentrique ", donc (d'un certain point de vue) conservateur. Mais on trouve cependant chez Wordsworth, dans la communion empathique et réciproque qu'il découvre entre le moi et le monde, l'origine d'une critique du dualisme inhérent au sujet cartésien, qui demeure un trait caractéristique essentiel et récurrent de l'écopoésie jusqu'à nos jours. La référence philosophique obligée de l'écodiscours qui à ce propos s'impose le plus fortement, c'est Merleau-Ponty et sa phénoménologie anti-cartésienne.

Dans la mesure où le logocentrisme est assimilé au phallocentrisme, la critique du sujet cartésien phallogocentrique est une question centrale chez les femmes poètes. Dans cette veine, Wordsworth fait par exemple l'objet de pages mémorables dans le Spéculum de l'autre fermme de Luce Irigaray. Le sujet dans son rapport au logos constitue un thème essentiel de l'écoféminisme. En l'occurrence, le terme " écoféminisme » fut inventé par Françoise d'Eaubonne dans Le Féminisme ou la mort (1980), où la destruction de la nature et l'oppression des femmes sont présentées comme un même travers de la civilisation occidentale. La façon dont cette vision des choses se rattache au volet antichrétien de l'écodiscours est assez évidente. Elle est fort bien illustrée dans un compte rendu de lecture de The Environmental Revolution de Max Nicholson (1970), où Hughes déclarait que "The subtly apotheosised misogyny of Reformed Christianity is proportionate to the fanatic rejection of Nature, and the result has been to exile Man from Mother Nature " (Hughes 129). Mais tout le problème, pour les écoféministes, réside précisément dans cette assimilation de la Femme à la Mère Nature, phénomène androcentrique, c'est-à-dire propre à l'imaginaire masculin. Le problème est très bien posé par Sherry B. Ortner, dans un article intitulé "Is female to male as nature is to culture? » (1974). Il existe néanmoins un deuxième temps de l'écoféminisme, constitué par la réalisation de ce que le refus même de situer la femme du côté de la nature revient précisément à perpétuer l'attitude androcentrique responsable de l'oppression de la femme comme de la destruction de la nature. Tel est le dilemme de l'écoféminisme. Il se retrouve, décliné de diverses manières, dans l'écopoésie féminine, par exemple sous des formes d'empathie écocentrique, dans des thématiques topophiles, ou bien dans des modes d'expression gynocentriques où domine ce que Julia Kristeva appelle le sémiotique, par contraste avec le sémiologique.

L'écopoésie, plus encore que l'écocritique, est un écheveau fait de nombreux fils. On en trouve des éléments sous la plume de tous les poètes, et il serait impossible de prétendre en faire exhaustivement le tour. Plus d'un poète reconnu a été évoqué au cours de ce rapide aperçu cartographique 
du paysage écocritique contemporain. Nombreux aussi sont les poètes d'aujourd'hui chez qui il faudrait relever des traits caractéristiques de cette tendance. Les Murray (né en 1938), John Burnside (né en 1955), Kathleen Jamie (née en 1962) et Alice Oswald (née en 1966) sont quatre exemples remarquables de ce courant poétique, dont les thèmes et les préoccupations caractéristiques se retrouvent chez nombre de poètes britanniques écrivant de nos jours. L'écopoésie est avant tout un discours poétique, exprimé de façons diffuses et à divers degrés d'importance chez tous les poètes britanniques au tournant $\mathrm{du} \mathrm{XX}{ }^{\mathrm{e}}$ siècle, exception faite, peut-être, de ceux qui se réclament des "Language poets » américains, dont les références philosophiques professent souvent un rejet radical de l'idéologie romantique. Mais encore, il n'est pas jusqu'à J. H. Prynne et ses compères de la «Cambridge School » chez qui puissent se surprendre des échos et des influences apparentés à ces problématiques. Certes, écopoésie et écocritique demeurent à bien des égards des inventions littéraires américaines, dont les poètes britanniques, certains plus que d'autres, paraissent accompagner le mouvement. Cependant, aux États-Unis l'écopoésie s'inscrit dans une continuité retrouvée avec le Transcendantalisme, dont le modernisme et le postmodernisme avaient tenté de faire leur deuil. Au Royaume-Uni, néanmoins, il se pourrait que la tradition romantique n'ait pas vraiment connu d'éclipse, par le fait de certaines figures comme W. B. Yeats ou G. M. Hopkins, puis Dylan Thomas, Ted Hughes et Seamus Heaney (pour ne citer que quelques noms très évidents) qui maintinrent le flambeau du romantisme d'un bout à l'autre de la terre-gâte technologique du XX $\mathrm{XX}^{\mathrm{e}}$ siècle. Mais tout au long de l'histoire de la littérature anglaise, depuis le plus haut moyen-âge, se repère une affection particulière pour les " Nature Poets ", dont Wordsworth fait un peu figure de parangon, et l'histoire des idées anglaises est parcourue par une fascination artistique et littéraire toujours bien vivace pour les rapports entre l'humain et l'inhumaine nature. Cela s'articule souvent avec la diversité culturelle de la nation britannique, et les rêveries primordialistes si chères aux anglais prennent des couleurs et tournures bien particulières à mesure qu'on s'éloigne un tant soit peu de Londres et des Lumières anglaise, depuis les «franges celtiques » d'Écosse, d'Irlande ou du Pays de Galles, jusqu'aux îles et régions les plus lointaines du Commonwealth. Ni américaine, ni britannique, l'écopoésie pose peutêtre bien le problème crucial de l'existence et de la survivance de la poésie aujourd'hui et demain, dans la littérature-monde. Car s'il existe bien une manière de littérature qu'il serait assez juste d'appeler industrielle, une techno-fiction en quelque sorte, il faut bien reconnaître que c'est une espèce dans laquelle les poèmes sont plus que rares. Enfin, la question reste ouverte de savoir s'il peut exister une poésie qui ne soit radicalement pas une écopoésie. Nous en pourrions peut-être confier la confection à nos ordinateurs, mais nous donnerions-nous encore la peine de la lire? 


\section{Bibliographie}

ASLE online (The Association for the Study of literature and Environment). http:// www.asle.umn.edu/

ISLE (Interdisciplinary Studies in Literature and Environment) http://www.unr. edu/cla/engl/isle/

Green Letters http://www.rlyeh.entadsl.com/ASLE/Green_Letters.htm

Abbs, Peter, ed. Earth Songs: A Resurgence anthology of contemporary poetry. Dartington Totnes: Green, 2002.

Adorno, Theodor and Rolf Tiedermann. Aesthetic Theory. 1970. London: Routledge, 1984.

Augé, Marc. Non-lieux. Introduction à une anthropologie de la surmodernité. Paris : Seuil, 1992.

Bachelard, Gaston. La Poétique de l'espace. [1958]. Paris : PUF, 2004.

Bate, Jonathan. Romantic Ecology: Wordsworth and the Environmental Tradition. London: Routledge, 1991.

- The Song of the Earth. London: Picador, 2000.

Brathwaite, Edward Kamau. History of the Voice: the Development of Nation Language in Anglophone Caribbean Poetry. London and Port of Spain, 1984.

-. The Arrivants. Oxford UP, 1973.

Bryson, J. Scott, ed. Ecopoetry. A Critical Introduction. Salt Lake City: U of Utah P, 2002.

Buell, Laurence. The Future of Environmental Criticism: Environmental Crisis and Literary Imagination. Oxford: Blackwell, 2005.

Burnside, John. Selected Poems: London: Jonathan Cape, 2006.

Césaire, Aimé. Une tempête. Paris : Seuil, 1969.

Chatwin, Bruce. The Songlines. [1987]. London: Picador, 1988.

Cohen, Michael P. "Blues in the Green: Ecocriticism Under Critique.” Environmental History 9.1. http://www.historycooperative.org/journals/eh/9.1/cohen.html 17 April 2007.

Cronon, William. "The Trouble with Wilderness; or, Getting Back to the Wrong Nature." Cronon, William, ed. Uncommon Ground: Rethinking the Human Place in Nature. New York: Norton., 1995. 69-90.

De Man, Paul. Blindness and Insight. Essays in the Rhetoric of Contemporary Criticism. [1971]. Saint Paul: U of Minnesota P, 1983.

Gifford, Terry. "Recent Critiques of Ecocriticism." Unpublished essay to appear in New Formations. 2007.

-. Green Voices; Understanding Contemporary Nature Poetry. Manchester: Manchester UP, 1995.

—. Pastoral. London: Routledge, 1999.

-. Reconnecting with John Muir: Essays in Postpastoral Practice. Athens: U of Georgia P, 2006.

—. "Gary Snyder and the Post-pastoral". Bryson, J. Scott, ed. Ecopoetry; A Critical Introduction. Salt Lake City: U of Utah P, 2002. 77-87.

Gilcrest, David W. Greening the Lyre: Environmental Poetics and Ethics. Reno: U of Nevada P, 2002.

Haar, Michel. Le Chant de la terre: Heidegger et les assises de l'histoire de l'Être. Paris : L'Herne, 1987.

Harrison, Robert Pogue. Forests: The Shadow of Civilization. Chicago: U of Chicago P, 1992.

Heidegger, Martin. Hölderlin's Hymnen 'Germanien' und 'Der Rhein'. Trans. Bruce V. Foltz. Inhabiting the Earth: Heidegger, Environmental Ethics, and the Metaphysics of Nature. New Jersey: Humanities P, 1995. 
—. "The Question Concerning Technology." Basic Writings. Ed. D.F. Krell. San Francisco: HarperSanFrancisco, 1993.

-. Poetry, Language, Thought. London: Harper and Row, 1971.

Hopkins, Gerard Manley. The Poems of Gerard Manley Hopkins. Oxford: Oxford UP, 1970.

Hughes, Ted. Winter Pollen: Occasional Prose. London: Faber, 1994.

Irigaray, Luce. Spéculum de l'autre femme. Paris : Minuit, 1974.

Jamie, Kathleen. Waterlight: Selected Poems. Saint Paul (MN): Graywolf P, 2007.

Killingsworth, M. Jimmie and Jacqueline S. Palmer. Ecospeak: Rhetoric and Environmental Politics in America. Carbondale: U of Illinois P, 1992.

Latour, Bruno. Nous n'avons jamais été modernes : essai d'anthropologie symétrique. Paris: La Découverte, 2006.

Liu, Alan. Wordsworth: the Sense of History. Stanford: Stanford UP, 1989.

Marx, Leo. "The Struggle over Thoreau”. New York Review of Books 46 (24 June 1999).

McKibben, Bill. The End of Nature. New York: Random House, 1989.

Merleau-Ponty, Maurice. L'Eil et l'Esprit. [1964]. Paris : Gallimard, 2006.

—. Phénoménologie de la perception. [1945]. Paris : Gallimard, 1976.

-. Le Visible et l'Invisible. Paris : Gallimard, 1964.

-. La Prose du Monde. Paris : Gallimard, 1969.

Meeker, Joseph. The Comedy of Survival: Literary Ecology and a Play Ethic. 1974. U of Arizona P, 1997.

Murray, Les. Learning Human: New Selected Poems. Manchester: Carcanet, 2001.

-. Translations form the Natural World. Manchester: Carcanet, 1992.

—. Persistence in Folly. Sydney and Melbourne: Angus and Robertson, 1984.

-. Dog Fox Field. London: Carcanet, 1991.

—. The Daylight Moon. London: Angus and Robertson, 1987.

- " "The enemy within." Review of New Collected Poems. The Guardian (15 March 2003). <http://www.carcanet.co.uk/cgi-bin/scribe? showdoc=281; doctype = review> 19 April 2007 .

Ortner, Sherry B. 1974. "Is female to male as nature is to culture?" M. Z. Rosaldo and L. Lamphere (eds), Woman, culture, and society. Stanford (CA): Stanford UP. 68-87.

Oswald, Alice. Dart. London: Faber, 2002.

-. Woods, etc. London: Faber, 2005.

—, ed. The Thunder Mutters: 101 Poems for the Planet. London: Faber, 2005.

Rousseau, Jean-Jacques. Discours sur les sciences et les arts. [1750]; Discours sur l'origine de l'inégalité. [1755]. Paris : Garnier-Flammarion, 1971.

Scigaj, Leonard. Sustainable Poetry; Four American Ecopoets. Lexington: UP of Kentucky, 1999.

Serres, Michel. Le Contrat naturel. Paris : Bourin, 1990.

—. Éclaircissements. Entretiens avec Bruno Latour. Paris : Bourin, 1992.

Teilhard de Chardin, Pierre. "Panthéisme et Christianisme ». Comment je crois. [1934]. Paris : Seuil, 1969.

—. La Place de l'homme dans la nature. [Albin Michel, 1956]. Paris : Seuil, 1965.

-. Etre plus. Paris : Seuil, 1968.

Weber, Max. L'Éthique protestante et l'esprit du capitalisme. [1904]. Paris: Flammarion, 1999.

White, Lynn Townsend Jr. “The Historical Roots of Our Ecologic Crisis.” Science. Vol. 155, number 3767 (March 10, 1967): 1203-207. http://www.earthtalktoday.tv/earthtalk-voices/historical-roots-ecological-crisis.html.

Zimmerman, Michael, E. Heidegger's Confrontation with Modernity: Technology, Politics, Art. Bloomington: Indiana UP, 1990. 